\title{
Pratiques
}

Linguistique, littérature, didactique

\section{Didactique et perspective historique. À propos d'une recherche sur les écritures de soi à l'école élémentaire}

\section{Marie-France Bishop}

\section{OpenEdition}

\section{Journals}

Édition électronique

URL : http://journals.openedition.org/pratiques/1568

DOI : $10.4000 /$ pratiques. 1568

ISSN : 2425-2042

Éditeur

Centre de recherche sur les médiations (CREM)

Édition imprimée

Date de publication : 15 juin 2010

Pagination : 231-248

Référence électronique

Marie-France Bishop, « Didactique et perspective historique. À propos d'une recherche sur les écritures de soi à l'école élémentaire », Pratiques [En ligne], 145-146 | 2010, mis en ligne le 15 juin 2010, consulté le 20 avril 2019. URL : http://journals.openedition.org/pratiques/1568 ; DOI : 10.4000/ pratiques. 1568 


\title{
Didactique et perspective historique. À propos d'une recherche sur les écritures de soi à l'école élémentaire
}

\author{
Marie-France Bishop \\ IUFM de Versailles, Université de Cergy-Pontoise
}

\begin{abstract}
"Faire l'histoire d'une discipline scolaire, c'est s'attacher non seulement aux programmes et aux finalités de la discipline, non seulement aux pratiques éducatives et enseignantes qu'elle met en œuvre, mais également aux effets réels et concrets qu'elle produit sur les élèves et la société qui les entoure. » (Chervel, 2006, p. 7).
\end{abstract}

Cette citation d'André Chervel définit le cadre dans lequel s'effectuent la plupart des recherches en didactique adoptant une visée historique. La démarche se retrouve dans les travaux analysant la discipline dans son ensemble (Jey, 1998 ; Houdart-Mérot, 1998 ; Chervel, 2006), ou ceux qui traitent des questions de corpus (Denizot, 2008), ou qui analysent les transformations d'un seul exercice comme la dictée, (Chervel, Manesse, 1989), pour ne citer que ces quelques exemples. Dans le cadre d'un travail de thèse, j'ai adopté ce canevas pour étudier un type de rédaction spécifique, les écritures de soi à l'école élémentaire de 1882 et 2002. L'étude, reprise dans cet article, a été réalisée en tenant compte des trois niveaux évoqués qui ont servi, en quelque sorte, de feuille de route aux différentes investigations et à la recherche de documents.

Le premier niveau est le cadre institutionnel tel qu'il apparaît dans les programmes et dans les différents textes officiels ou semi-officiels. Il s'agit de la définition des objectifs, des finalités et de l'ensemble des préconisations. Mais ce palier est très large car il croise des données institutionnelles, sociales, politiques. La compréhension des enjeux ne peut se cantonner aux seuls textes officiels et doit prendre appui sur des ouvrages d'histoire de l'éducation. Le second niveau est celui des pratiques conseillées qui se découvrent dans la consultation des manuels, des revues professionnelles et aussi grâce aux échanges entre inspecteurs et enseignants, comme lors des conférences pédagogiques. Ce second ensemble est celui des documents qui définissent les contenus à enseigner, ce sont des prescriptions secondaires qui correspondent en partie aux pratiques réelles puisqu'elles mettent en relation la demande officielle et le concret des situations scolaires. Enfin, le troisième niveau est sans doute le plus complexe à aborder. En effet, comment découvrir les répercussions réelles de la discipline, si ce n'est en observant directement le travail 
des élèves ou en se fiant à des témoignages ? Pour cela, la consultation de cahiers d'élèves conservés dans les musées ${ }^{(1)}$ à été nécessaire, ainsi que le recours aux écrivains et à leurs souvenirs d'écolier.

Mais la perspective historique nécessite aussi de prendre en compte les questions spécifiques à la didactique, comme la délimitation de l'objet sur lequel on souhaite travailler. En effet, que faut-il entendre par «écritures de soi »? cette dénomination est-elle pertinente à l'école ? convient-elle à toutes les époques? Un autre problème consiste à faire coïncider les grands moments de l'histoire scolaire avec la périodisation particulière liée aux transformations de l'objet étudié, les temporalités n'étant pas toutes identiques. C'est ainsi que la double inscription, à la fois historique et didactique nécessite de tenir compte d'une diversité de contraintes, au risque parfois d'une certaine hétérogénéité.

\section{Les écritures de soi, une pratique scolaire à interroger}

\section{Un exercice ambigu}

Les écritures de soi existent à l'école depuis longtemps, sous des formes variées, même si elles ne s'affichent que rarement en tant que telles. Elles prennent appuient sur le vécu des élèves et nécessitent l'emploi de la première personne. En voici la prescription dans les instructions de 2004, pour la classe de sixième :

«Le récit qui s'appuie sur l'expérience personnelle sera également pratiqué. Il fait de la première personne la personne de référence, situation d'énonciation qui peut entraîner des problèmes rédactionnels propres (emploi des temps verbaux par exemple). Il exige aussi, en raison de sa proximité avec les événements réels, un effort de construction particulier. Mais il présente l'avantage, du fait même qu'il la prend en compte par l'écriture, de donner toute sa dignité à l'expérience vécue par l'élève, quelle qu'elle soit. ».

Cet exemple est emblématique de l'ambiguïté de ces écritures. Mal définies au niveau générique, elles sont perçues comme complexes et cependant intéressantes car agissant au niveau de l'individu, soit en lui redonnant toute sa dignité, soit en lui permettant de se (re)construire. Cette situation est problématique, car l'école demande à l'élève de se raconter tout en l'évaluant sur la manière dont il le fait mais sans le lui avoir enseigné. Actuellement, une confusion semble s'établir entre écriture de soi scolaire et écriture autobiographique, comme si la seule incitation à parler de soi suffisait à placer le scripteur dans une position volontaire d'autobiographe. Philippe Lejeune s'est intéressé à l'engagement intentionnel de l'écrivain qui décide de raconter sa vie, ce qu'il a désigné sous le nom de «pacte autobiographique ${ }^{(2)}$. Il définit, par ce concept, les différentes formes de déclaration d'intention de l'écrivain qui s'engage de manière plus ou moins explicite à produire un récit véridique de sa vie. Pacte réel ou illusoire, il repose dans tous les cas sur la libre détermination de son auteur: "Une autobiographie, ce n'est pas quelqu'un qui dit la vérité sur lui-même, mais quelqu'un qui dit qu'il la dit. " ${ }^{(3)}$ Mais dans le cadre de l'école, ce pacte ne peut exister. L'élève ne s'engage pas à tenir un discours de vérité, il cherche seulement à répondre à une consigne donnée, en espérant s'approcher le

(1) Et plus particulièrement au Musée de Saint-Ouen l'Aumône dans le Val d'Oise qui dispose de belles collections de documents scolaires.

(2) Philippe Lejeune, L'autobiographie en France, Paris, Armand Colin, 1971.

(3) Philippe Lejeune, Les Brouillons de soi, Paris, Seuil, 1998, p. 125. 
plus possible du modèle qui servira de référence lors de l'évaluation. Certes, il y a concordance entre le personnage, le narrateur et l'auteur, mais ce point est le seul qui soit identique entre le contrat scolaire et le contrat autobiographique littéraire. Le contrat scolaire, dans le cas des écritures de soi, est paradoxal ${ }^{(4)}$ car il impose à l'élève de produire un récit qui ne sera jugé que selon sa conformité à l'attendu scolaire. Ce qui fait que toute production écrite est le fruit d'un calcul de l'élève consistant à rechercher la stratégie la plus efficace possible. Ce dernier n'écrit pas ce qu'il veut, mais ce qu'il pense être attendu par le maitre.

La pratique de l'écriture de soi scolaire est prise dans cette tension entre expression de soi et obligation scolaire, entre "dignité de l'expérience personnelle » (selon les Programmes de $6^{\mathrm{e}}$ cités) et apprentissage de la langue, entre construction de l'individu et évaluation.

Face à cette ambiguïté, l'élève peut, s'il a perçu les usages scolaires implicites, adopter une position distanciée. Mais, à l'inverse, certains commettent une véritable erreur d'interprétation consistant à prendre la demande autobiographique « au pied de la lettre » ${ }^{(5)}$, c'est-à-dire comme une invitation à dire quelque chose de vrai et de personnel. Dans ce cas, l'injonction peut être ressentie comme une intrusion à laquelle l'élève oppose un refus catégorique, comme dans cet exemple daté de 2001, provenant d'une classe de CM1 où il avait été demandé de raconter un événement vécu. Voici la réponse d'un jeune élèvedans l'orthographe d'origine : "Je ne veux pas vous raconter ma vie parce que sa ne vous regarde pas ". À l'opposé, la sollicitation peut conduire à des déversements qui sortent du cadre scolaire et qui laissent l'enseignant désemparé, comme dans ce texte provenant de la même classe de CM1 :

" J'ai 9 ans édemie je mapaille P-E, à 2 ans dans la nuit ma maman avait divorsé. Je demander à mon père ou ma mère était il disè qu'elle était au travail. Et mon père ne me disait pas la vérité. Et plus tard mon père mavait dit que ma mère avait divorsé. J'ai pleré. Et 1 ou 2 ans après mon père rencontra sonia et, après on à déménagé. »

Cette difficulté que rencontre l'expression de la subjectivité dans le cadre scolaire est liée à l'absence de définition de ces écritures particulières qui sont rarement l'objet d'un apprentissage.

\section{Des pratiques mal définies}

Les écritures de soi sont difficiles à définir, elles hésitent entre deux pôles extrêmes. Le premier, déjà évoqué, est celui des genres autobiographiques. Les écritures de soi désignent, dans ce cas, un ensemble de genres littéraires organisés à partir de caractéristiques particulières et incontournables, comme Philippe Lejeune 1'a proposé pour l'autobiographie ou le journal intime ${ }^{(6)}$. Cette approche, qui détermine des points communs et délimite des catégories, peut également s'avérer restrictive, car il y a toujours des variations pour lesquelles se pose la question de l'appartenance au genre. Mais, dans tous les cas, l'approche générique est confortable car elle facilite les classements et fourni des éléments de reconnaissance stables et re-

(4) Cette analyse renvoie aux travaux de Jean-François Halté sur la question du « malentendu »: «L'annotation des copies et l'évaluation », dans Pratiques n 44, 1984 ; «Travailler en projet » dans Pratiques $n^{\circ} 36,1982$.

(5) Voir les analyses de Bernard Lahire qui associe ces erreurs d'interprétation des demandes scolaires à l'appartenance sociale des élèves. B. Lahire, Culture écrite et inégalités scolaires, Lyon, Presses universitaires de Lyon, 1993.

(6) Philippe Lejeune, Catherine Bogaert, Un journal à soi, Paris, textuel, 2003. 
productibles. Avec l'introduction du biographique comme objet d'étude ${ }^{(7)}$, l'école s'en est largement emparée, créant une confusion entre écritures de soi et autobiographie.

À l'opposé, les écritures de soi peuvent apparaître comme extrêmement fluides, présentes dans toute expression de la subjectivité de l'auteur. Dans ce sens, chaque manifestation du moi, détournée, suggérée ou seulement effleurée est une écriture de soi, dont rien n'indique la présence, ni les marques linguistiques, ni les contenus sémantiques. L'écriture de soi se découvre, alors, par la présence d'éléments subtils, par un bruissement du subjectif. Ce peut être là où on l'attendait le moins qu'advient l'expression de soi qui ne peut être perçue que par l'interprétation du lecteur ${ }^{(8)}$. S'il est indéniable que nombre d'écrits manifestent la subjectivité de leur auteur, l'extrême labilité des formes empêche de saisir les écritures de soi comme objet.

Pour éviter ces deux extrêmes, cadre générique littéraire ou omniprésence de la subjectivité, les écritures de soi sont abordées, dans le cadre de cette étude, comme un objet scolaire, c'est-à-dire répondant à des critères pédagogiques spécifiques. Tout d'abord, il s'agit d'écritures produites pour l'école, avec toutes les particularités liées à ce cadre institutionnel précis. Se trouvent alors exclues, les pratiques relevant du domaine de la littérature générale, mais aussi les écritures extrascolaires des écoliers (M.-C. Penloup, 1999). Les écritures de soi doivent être reconnaissables, donc porter des marques linguistiques et énonciatives précises. Sans nier la présence possible du subjectif dans tout écrit, ces marques sont indispensables pour délimiter une modalité d'écriture spécifique qui utilise le « je » comme dénotation du sujet scripteur, associé à un contenu faisant référence à des éléments de la vie de l'élève-auteur. Se trouvent ainsi écartés les écritures de fictions et les emplois de «je» fictifs qui peuvent manifester la subjectivité du scripteur, mais ne constituent pas (au sens que nous leur donnons ici) des écritures de soi. La définition qui semble le mieux convenir à cet objet scolaire qui commence à être cerné est celle de Georges Gusdorf qui reconnaît les écritures de soi dans : " ...tous les cas où le sujet humain se prend lui-même pour objet du texte qu'il écrit. La littérature du moi, en sa plus vaste ampleur, est l'écriture du "je", destinée à autrui ou réservée à la consommation personnelle. Encore faut-il que la première personne ainsi utilisée ne soit pas seulement une fiction grammaticale ou un procédé rhétorique. " ${ }^{(9)}$

L'intérêt de cette définition est son amplitude au-delà des marques génériques textuelles, ce qui va permettre d'intégrer tous les types d'écrits utilisant un « je » déictique et évoquant des éléments liés directement à l'auteur et/ou à sa vie. Ce dernier point constitue la grande particularité de cet objet scolaire qui met en scène le sujet lui-même. La relation entre écriture scolaire et subjectivité va sans cesse être redéfinie en fonction des conceptions de l'individu et de son éducation que la société et l'école ont développées. C'est en cela que les variations historiques des écritures de soi, qui sont directement liées aux finalités éducatives des différentes époques, constituent un versant indissociable de l'analyse didactique.

(7) Le biographique apparaît dans les programmes de Première de 2001 et disparaît en 2006.

(8) Se retrouvent ici les positions de Frédéric François qui revendique une lecture interprétative des récits d'enfants. Frédéric François, Enfants et récits, Villeneuve d'Ascq, Presses Universitaires du septentrion, 2004.

(9) G. Gusdorf, (1991), Ligne de vie-Les écritures du moi, Paris : éd. Odile Jacob, p. 122. 


\section{Naissance et apogée des écritures de soi (1882-1958)}

\section{«Des sujets se rapportant à la vie personnelle de l'enfant"}

L'histoire des écritures de soi se confond avec celle de la mise en place de la rédaction dans le primaire, sous la troisième République. Au début de la décennie de 1880 , sous l'influence des pédagogues du parti républicain, la rédaction est introduite de manière officielle, comme nouvel exercice de français à l'école primaire, dans l'arrêté du 27 juillet 1882, définissant « l'organisation pédagogique et le plan d'études des écoles primaires ». Cet acte de naissance constitue une alternative à l'enseignement traditionnel de la langue fondé sur la mémorisation et la récitation des règles de grammaire (Boutan, 1996 ; Chervel, 2006). Déjà sous le Second empire, des critiques sévères s'élèvent contre la méthode traditionnelle et certains membres influents de l'administration y font écho, tels Victor Duruy ministre de l'instruction et Octave Gréard directeur de l'Instruction primaire de la Seine. Ce dernier, recommande aux maîtres de son département, dès $1868^{(10)}$, de prendre appui sur ce que les élèves connaissent de la langue en faisant écrire des petits textes aux écoliers. Le principe d'utiliser l'écriture pour apprendre la langue est généralisé en juillet 1882, dans les programmes de l'école primaire puisque dès le cours moyen on propose : « les Premiers exercices de rédactions sur les sujets les plus simples et les mieux connus des enfants " ${ }^{(11)}$. La rédaction s'installe sous cette forme pour quatre-vingt-dix ans. Les Instructions de 1923 et celles de 1938 n'en modifient pas la substance puisque pendant toute la première partie du $\mathrm{XX}^{\mathrm{e}}$ siècle, on va proposer aux élèves d'écrire sur : "un sujet simple se rapportant à la vie personnelle de l'enfant (scolaire ou familiale) " ${ }^{(12)}$.

Quels sont ces « sujets simples, se rapportant à la vie personnelle»? Les manuels, les revues pédagogiques et divers ouvrages à l'usage des maîtres permettent de découvrir ce qui était proposé dans les classes. Ainsi, la librairie Hachette qui publie les Instructions de $1923^{(13)}$ les accompagne de listes de sujets de rédactions et de récitation, fort utiles pour connaître les supports utilisés. La consultation des quarante sujets de rédaction proposés pour le cours moyen deuxième année conduit à différents constats. Le premier est qu'un peu plus du quart des sujets s'adressent de manière explicite à l'élève et sollicite des éléments de la vie personnelle par un « vous » injonctif, comme dans cet exemple :

"Racontez le jour de la rentrée, en analysant vos sentiments : l'école repeinte; les tableaux plus noirs ; des camarades partis ; des nouveaux ; le plaisir de se retrouver ; la classe paraît agréable. ».

La seconde remarque est que la moitié des intitulés évoquent des situations de la vie quotidienne, sans injonction autobiographique, comme dans cet exemple: «Les premiers pas de bébé. Les encouragements et l'aide de la maman; la chute; maman console et sourit. "Recommençons! » La troisième remarque est que moins du quart est constitué de sujets faisant appelà l'imagination des élèves, l'école républicaine manifestant une grande méfiance face à l'imagination enfantine. Cet emploi massif

(10) 1868, Organisation pédagogique des écoles du département de la Seine.

(11) André Chervel, L'enseignement du français à l'école élémentaire, tome 2., Paris, INRP, 1995 , p. 105.

(12) « 23 mars 1938 - Arrêté modifiant le règlement du certificat d'études primaires », dans André Chervel, op. cit., t. II, p. 362.

(13) Programmes officiels des Écoles primaires élémentaires, Paris, Librairie Hachette, 1923 ( $2^{\mathrm{e}}$ édition, 1924). 
des sujets faisant appel à l'expérience vécue des élèves va se confirmer sous les III ${ }^{\mathrm{e}}$ et IV ${ }^{\mathrm{e}}$ Républiques, créant un véritable phénomène que renforce l'usage important de ce type de sujets dans les rédactions du certificat d'études primaires. Ouvrons par exemple un manuel de préparation au $\mathrm{CEP}^{(14)}$, édité de nombreuses fois entre 1954 et 1980 : la proportion des demandes d'écriture fondée sur l'expérience et utilisant la première personne dépasse largement la moitié des sujets proposés. Dans ce même manuel, se rencontrent moins de dix sujets d'imagination, et la référence au monde quotidien est constante. Ce trait particulier des sujets de rédactions fondés sur des éléments vécus connaît une belle longévité et va même devenir emblématique des rédactions de cette époque. On peut s'interroger sur les raisons de cette utilisation importante des écritures de soi à l'école élémentaire, et revenir, pour mieux les comprendre, sur les finalités attachées à cet exercice et sur les principes éducatifs mis en place sous la III $^{\mathrm{e}}$ République.

\section{Les enjeux des écritures de soi}

L'école de la III ${ }^{\mathrm{e}}$ République est soumise à deux principes divergents ${ }^{(15)}$ qui se trouvent en tension dans la rédaction et surtout dans la sollicitation récurrente du vécu. La première tendance relève d'un souci d'éducation populaire et de rénovation pédagogique portée par certains républicains, dont les plus célèbres sont Ferdinand Buisson, Octave Gréard et bien d'autres encore. Il s'agit d'une aspiration à la mise en place d'un enseignement spécifique au primaire, fondé sur la méthode intuitive et l'observation, dont la leçon de choses est l'illustration (Kahn, 2002). Cette pédagogie prône l'activité de l'élève, elle est inspirée par Rousseau et les pédagogues suisses de la fin du XVIII ${ }^{\mathrm{e}}$ siècle. Mais une seconde tendance, relevant de l'idéologie politique, est celle d'un conservatisme social qui est encore bien présent dans l'action des Républicains, lesquels sont soucieux du maintien de l'ordre social après les crises politiques du XIX ${ }^{\mathrm{e}}$ siècle et la Commune de Paris de 1871. Le but est de maintenir une organisation sociale stable fondée sur une répartition traditionnelle des rôles sociaux. Pour cela on met en place une éducation autoritaire visant cependant l'autonomie des personnes par le droit civique ${ }^{(16)}$. Cette éducation est fondée sur l'inculcation morale et sur la fidélité à la patrie (Deloye, 1994).

La rédaction porte les traces de cette double aspiration, d'un côté, il s'agit de fonder l'apprentissage de la langue sur l'activité et l'écriture individuelle, mais d'autre part, on utilise l'écriture comme élément d'édification morale et patriotique. De même, s'il faut donner aux enfants des classes populaires la maîtrise écrite du français, il ne peut être question d'excéder le cadre pratique de l'enseignement élémentaire. C'est en tenant compte de ces deux aspirations que va être envisagée la rédaction pour les élèves des écoles communales. La solution la plus accessible est de prendre appui sur ce qui est à leur portée, sans avoir recours à la littérature classique qui est le domaine réservé du secondaire, c'est-à-dire d'utiliser ce qu'ils connaissent : leur vécu et leur quotidien.

Mais ces rédactions sollicitant le vécu n'ont pas de visée autobiographique, au

(14) Claude Michel et Louis Hubert, Guide pratique du Certificat d'études primaires, Paris, Bordas, 1956 ( $1^{\text {re }}$ édition).

(15) L'ouvrage de Christian Nique et Claude Lelièvre, La République n'éduquera plus. La fin du mythe Ferry, Paris, Plon, 1993, développe l'hypothèse d'une visée plus conservatrice qu'éducative dans l'œuvre scolaire de Jules Ferry et de la III $^{\mathrm{e}}$ République, tandis que Pierre Kahn, soutient l'idée d'une ambition culturelle dans les programmes de Jules Ferry. Pierre Kahn, La leçon de choses, Villeneuve d'Ascq, 2002.

(16) Marcel Gauchet, La démocratie contre elle-même, Paris, Gallimard, 2002. 
sens où nous l'entendons aujourd'hui. Leur but n'est pas l'expression du sujet. Elles répondent à un constant souci d'édification morale. Plutôt que de raconter de véritables souvenirs, on attend des élèves qu'ils écrivent des scènes élaborées à partir de schémas transmis par la littérature et appris grâce aux récitations ${ }^{(17)}$. Les récits sont cadrés par des consignes très directives, ce sont des sortes de scénarios prédéfinis, imprégnés de bons sentiments. On trouve par exemple, le scénario de la veillée en famille ${ }^{(18)}$, celui du jour de la rentrée, celui de la charité, de l'aveu de la faute, etc. Tous correspondent à des stéréotypes de la littérature scolaire et permettent de conforter une éducation civique et morale.

Les cahiers d'élèves conservés dans les Musées de l'Éducation et plus particulièrement celui de Saint-Ouen l'Aumône (95), ont permis de constituer un corpus important de travaux d'élèves pour cette période. Les annotations des maîtres et les corrections demandées aux élèves offrent des indications précieuses pour saisir les nuances entre demande explicite de l'intitulé et attendu implicite de l'institution. Le décalage entre ces deux niveaux est particulièrement marqué dans le cas des écritures de soi, car s'il est demandé explicitement à l'élève de se référer à des événements vécus ou supposés tels, il n'est jamais attendu de réels souvenirs. Voici l'exemple d'une rédaction provenant d'un cahier de cours moyen. Le devoir est daté du 18 février 1925 :

« Un homme âgé de 40 ans environ, fort et robuste est passé hier dans le village, un sac sur l'épaule et demandant l'aumône. Votre père qui est très charitable l'a fait causer un moment puis à refuser de le secourir. Dites pourquoi.

Développement.

Hier, il est passé dans mon village, un homme fort et robuste qui est venu demander l'aumône.

- Est-ce que vous avez une maison, lui demanda mon père?

- Oui, mais je l'ai vendue.

- Vous n'êtes pourtant pas infirme

- Non, mais quand mon père mourut, je n'étais pas vieux et j'étais pauvre.

- Il fallait aller dans un hopitathospice, quand vous auriez été assez grand, vous auriez pris un métier, et vous ne seriez pas mendiant.

- J'ai essayé de travailler, mais je n'étais pas assez fort.

- Vous n'avez pourtant pas l'air faible. Vous êtes donc un paresseux. Je ne vais pas vous faire l'aumône. Si vous vouliez vous ne seriez pas à demander aux portes votre nourriture. Allez-vous en ailleurs, je ne donne pas du pain aux fainéants, et je netes vous plains pas. C'est vous qui voulait ez bien être comme-ȩa ainsi. »

Ce devoir a été noté 6/10. Les remarques qui l'accompagnent, signalent quelques éléments mal dits et ajoutent ce commentaire final :

« Vous avez exagéré. Dans votre devoir, la preuve n'existe pas que l'homme est pauvre par sa faute. Vous donnez à votre père un cœur dur qu'il n'a certainement pas. ».

La remarque du maitre met en lumière les deux aspects du devoir. Le premier est la conformité avec un scénario lu et étudié, celui de la valeur du travail qu'il ne faut pas confondre avec celui de la charité souvent rencontré dans les rédactions. Le début de l'annotation évalue le récit de l'élève, non par rapport à un événement vécu

(17) C'est le récit que font Marcel Arland, Nathalie Sarraute, Claude Duneton et récemment Mona Ozouf, à propos des rédactions de leur enfance.

(18) Marie-France Bishop, « Le récit de la veillée en famille. Un exemple de la sollicitation du vécu dans les rédactions scolaires », Recherches, n45, 2006, pp. 123-134. 
qui n'a jamais été vraiment demandé, mais pour sa conformité au scénario : il fallait prouver que l'homme était pauvre par son manque de courage et trouver le ton juste pour le lui dire, ce que l'élève n'a visiblement pas su faire. La seconde partie de l'annotation manifeste un mouvement de rétroaction que 1'on remarque quelquefois dans les devoirs de la même époque ${ }^{(19)}$. En effet, bien que l'aventure ne soit pas supposée avoir été vécue, c'est malgré tout l'élève, en tant que sujet moral, qui est jugé à travers son écrit dans son amour filial.

La lecture de ces différents documents, manuels ou travaux d'élèves, renseigne sur les enjeux des écritures de soi au cours de cette première période. Il s'agit, d'une part, d'une éducation linguistique qui favorise l'acquisition d'un français scolaire, d'autre part, ces écritures sont mises au service de l'acquisition des valeurs morales et civiques, grâce à la réécriture du vécu sous forme de scénarios. Ce vécu raconté est un vécu prescrit, l'authenticité n'est pas l'objectif poursuivi. La visée éducative est très présente durant les premières années du $\mathrm{XX}^{\mathrm{e}}$ siècle. Elle va s'atténuer puis disparaître peu à peu après la Seconde Guerre mondiale, à mesure que les finalités éducatives de l'école se transforment, en lien avec les évolutions de la société.

\section{Le texte libre dans la pédagogie Freinet}

\section{Définition et enjeux des textes libres}

Mais le modèle traditionnel de la rédaction instauré en 1882 est l'objet de fortes critiques émanant, dès le début du $\mathrm{XX}^{\mathrm{e}}$ siècle, des mouvements d'éducation nouvelle dont le GFEN ${ }^{(20)}$. Freinet, proche de ce courant, s'insurge contre l'emprise moralisatrice et contraignante de l'école. Pour y échapper, il met en place une pédagogie centrée sur la libre expression, sur le travail et sur les activités collectives.

Le texte libre est l'emblème de cette démarche. C'est entre 1920 et 1925, à l'école primaire de Bar-sur-Loup, son premier poste, que cette technique voit le jour. Le jeune maître a découvert l'intérêt suscité par le récit d'un événement vécu collectivement. Pour conserver trace de ces écrits éphémères qui relatent des épisodes de la vie des élèves ou de la classe, il installe une imprimerie selon ce qu'il a vu faire dans l'est de l'Europe. Sans doute Freinet n'a-t-il pas inventé le texte libre, mais il va lui donner un sens particulier et l'attacher définitivement à son nom en le définissant comme une réponse à un besoin d'expression spontanée, authentique et libre :

"Le texte libre doit être vraiment libre, c'est-à-dire qu'on l'écrit lorsqu'on a quelque chose à dire, lorsqu'on éprouve le besoin d'exprimer, par la plume ou le dessin, ce qui bouillonne en nous. L'enfant écrira son texte spontané sur un coin de la table le soir ; sur ses genoux, en écoutant parler la grand-mère qui ressuscite pour lui les histoires étonnantes du temps passé ; sur le cartable, avant d'entrer en classe et aussi, naturellement, pendant les heures de travail libre que nous réservons dans notre emploi du temps».

Certains de ces textes sont écrits à la première personne et relatent des événements de la vie quotidienne et personnelle, ils peuvent, à ce titre, être rangés dans la catégorie des écritures de soi scolaires.

Les enjeux des textes libres sont multiples. Il ne s'agit pas seulement d'un moment de liberté et d'expression, mais d'un véritable outil pédagogique. En effet, ils sont au cœur de toute la pédagogie coopérative, puisqu'en donnant la parole aux

(19) Marie-France Bishop, «Les annotations, indicateurs des fonctions de la rédaction : parcours historique », Repères $n^{\circ} 31$, novembre 2005, pp. 9-27.

(20) Groupe français d'éducation nouvelle. 
élèves, le maître découvre les thèmes qui les intéressent et à partir desquels il élaborera le programme de la classe. De plus, ces textes ont une valeur psychologique, car ils offrent l'occasion de « restaurer l'unité de l'enfant» selon l'expression de Freinet. En effet, refusant les pratiques scolaires traditionnelles qui séparent les différents univers, le scolaire et le privé, Freinet préconise la libre expression dans la classe. Grâce aux textes libres, la vie entre dans l'école et l'élève retrouve cette unité qui seule peut stimuler son désir d'apprendre et lui permettre de se développer harmonieusement. Ensuite, ces textes répondent à des enjeux de socialisation, car ils n'ont de sens que dans un circuit de communication fondamental dans la pensée pédagogique de Freinet. Tout texte doit être choisi, repris et édité pour être envoyé aux correspondants ou figurer dans le journal de la classe. Ces écritures ne sont donc pas intimes, mais destinées à la communication et à la socialisation. Enfin ces écrits ont une fonction politique clairement affirmée ${ }^{(21)}$. Freinet entend faire de son école une véritable école prolétarienne et redonner, grâce aux textes libres, la parole aux enfants du peuple. Pour lui, l'école traditionnelle entretient le clivage entre bourgeoisie et prolétariat en privant ce dernier du véritable moyen d'expression qu'est l'écriture. L'école du peuple qu'il entend développer doit permettre aux enfants des classes populaires d'être capables de parler en leur nom, contrairement à ce qui leur est traditionnellement enseigné :

"C'est ainsi que l'école [traditionnelle], malgré tous ses efforts, prépare une masse d'enfants analphabètes parce que, bien que sachant lire et écrire, ils sont incapables d'exprimer par la plume les difficultés de leur vie, leurs joies, leurs peines, leurs rêves. Ils ont besoin que des étrangers à leur milieu traduisent, en les trahissant plus ou moins, leurs propres sentiments. " ${ }^{(22)}$

Car c'est bien l'écriture autobiographique qui est désignée comme étant le véritable mode d'expression des classes populaires. En cela, Freinet se rapproche des thèses du groupe des «Écrivains prolétariens » réunis autour d'Henri Poulaille qui définit l'écrivain prolétarien comme celui qui trouve son inspiration dans le vécu : "Sa source n'est pas la lecture, ni l'école, c'est la vie, la sienne et celle des autres, celle qu'il voit, qu'il observe ou qu'il vit. " ${ }^{(23)}$ Il ne s'agit pas d'une écriture de soi introspective (l'auteur honni est Proust), mais d'une écriture très proche du témoignage, d'une écriture qui manifeste la cohésion d'une classe sociale et lui donne l'occasion de se construire une identité collective.

\section{Les particularités des textes libres}

Destinés à être envoyés aux correspondants ou à être imprimés dans le journal, les textes libres, qui servent également de supports de lecture, présentent des traits récurrents. Leurs phrases sont brèves, ils ne contiennent que peu d'adverbes ou d'adjectifs, de ce fait, ils sont faiblement modalisés, comme si aucune évaluation de l'action n'était attendue. Les faits sont rapportés dans leur succession, sans que le jeune narrateur n'exprime de sentiments, contrairement aux écrits des classes traditionnelles dans lesquels il était demandé d'exposer le ressenti. En voici un exemple caractéristique, provenant d'une classe de Château-Chinon et rédigé en $1947^{(24)}$ :

(21) Selon 1'hypothèse extrêmement intéressante de Marie-Hélène Vourzay dans sa thèse, Cinq discours sur la rédaction (1870-1989), thèse pour le doctorat, université de Lyon, 1996.

(22) Célestin Freinet, La méthode naturelle de lecture, Verviers- Marabout, 1968, p. 353.

(23) Henri Poulaille, Nouvel âge littéraire, Paris, Librairie Valois, 1930, p. 101.

(24) Ces textes sont conservés au Musée de l'Education de Saint-Ouen l'Aumône (Val d'Oise), qui en a aimablement autorisé la reproduction. 
«Une partie de traîneau

Il neige. Avec mes camarades, nous fabriquons un traîneau. Nous partons pour le pré, en face la maison de Madame Chevrel. Nous attachons une corde à l'avant. Je monte. Charles tire. La ficelle casse. Je tombe et je roule dans un trou. Je me fais une grosse bosse à la tête. Je me ramasse. Je suis couverte de neige. Nous rions.

Mauricette»

L'élément qui peut paraître le plus marquant est certainement l'emploi du pronom du pluriel, «nous ». Celui-ci est la marque du collectif dans lequel le narrateur se fond. Cette fusion de l'individuel au sein du groupe est sensible dans ces glissements répétés $d u$ « je » au «nous ». Le narrateur n'apparaît souvent que comme un élément de la communauté dont il ne se sépare pas. En voici une illustration dans cet écrit daté de 1947 et rédigé dans une classe pratiquant la pédagogie Freinet à Château-Chinon :

«Veillée de Noël - A Noël, je me suis bien amusée. Nous avons bu du chocolat. René a récité. Didi a été gentille. Le Père Noël a chanté. Il a distribué des bonbons. Le sapin était garni de guirlandes. (Annick). »

À l'opposé, le « je » peut être utilisé dans un texte signé collectivement qui rapporte toutefois une aventure strictement individuelle, comme dans de nombreux récits de rêve. Voici le récit d'une promenade, écrit en 1951, provenant d'une classe Freinet de Frimainville :

\section{« Le printemps}

Hier matin, en montant aux Fiottes, j'ai entendu siffler le merle de la sapinière. Puis un papillon jaune vint s'amuser sur l'oreille de mon âne. En ramassant un pissenlit, j'ai trouvé un grillon qui chantait. Je l'ai pris dans la main pour le regarder. Mais hop ! il a sauté dans la haie. Je l'ai poursuivi en enfonçant mon doigt dans son trou noir. La terre était humide et chaude. J'ai cueilli un bouquet de primevères pour orner la voiture. J'avais envie de chanter "la Violette et le gai Printemps ! Les lions".»

La signature collective de cette promenade printanière et presque amoureuse, tissée de petits détails est paradoxale. Elle met en valeur la fusion entre l'individu et le groupe, lors de la rédaction des textes libres. Car le véritable sujet est la classe ou le groupe, c'est-à-dire un sujet collectif, fortement structuré autour de rituels comme l'écriture, la correspondance et surtout la réécriture collective des textes. Ce collectif a une valeur politique, puisqu'il permet l'incorporation de l'individu dans la structure sociale. Mais, de nouveau, l'écriture de soi est marquée par une ambiguïté liée aux conditions de sa production, puisqu'elle échappe à l'individu pour se fondre dans une entité collective.

Les initiatives de Célestin Freinet et les thèses de l'Éducation nouvelle vont gagner en influence et étendre leur audience auprès de certains inspecteurs généraux et même de certains membres du ministère, au milieu du $X X^{\mathrm{e}}$ siècle. C'est ce courant de pensée qui est à l'origine de la rénovation du français de la fin des années soixante.

\section{La rénovation du français et le changement de perspective}

\section{La communication et l'expression de soi}

$\mathrm{Au}$ début de la $\mathrm{V}^{\mathrm{e}}$ République, l'édifice scolaire français connaît de profonds bouleversements. Un mouvement de réflexion sur l'enseignement du français dans le primaire s'amorce à la suite des réformes Berthoin de 1959 et Capelle-Fouchet de 1963, qui suppriment la scission entre les ordres scolaires et modifient les finalités 
de l'école élémentaire. L'âge obligatoire de la scolarité est reculé à 16 ans, à partir de 1967, avec une poursuite théorique des études dans le second degré. Ces changements nécessitent de repenser l'articulation entre la fin du cours moyen et la sixième. De plus, les difficultés des élèves conduisent les décideurs à envisager de transformer les programmes de l'école élémentaire, ce projet est confié à la commission Rouchette, en 1963, par Jean Capelle, directeur général des programmes scolaires et René Haby, sous-directeur des études. Avec l'accord du ministre Olivier Guichard, les propositions de la Commission Rouchette sont expérimentées autour d'écoles normales volontaires à partir de 1967, avec un pilotage de l'IPN (actuel INRP ${ }^{(25)}$ ) confié à Louis Legrand puis, en 1968, à Hélène Romian. Un document programmatique, le Plan de Rénovation, est élaboré collectivement en 1969. Mais ce Plan, fortement rénovateur et progressiste, dérange tant les politiques que les milieux conservateurs de l'enseignement, sa diffusion est freinée par le cabinet du président Georges Pompidou. Finalement, il ne sera publié qu'en 1971, après une campagne de dénigrement orchestrée par l'UNI ${ }^{(26)}$, syndicat étudiant de droite et quelques journaux comme Le Figaro ou La Revue des deux Mondes. Pour calmer les esprits, le ministère publie, en décembre 1972, des instructions officielles qui édulcorent une partie des propositions du Plan.

Malgré ce parcours chaotique et mouvementé, la rénovation est bien réelle et l'enseignement du français à l'école élémentaire change de configuration ${ }^{(27)}$. L'objectif de l'apprentissage est désormais la communication et l'utilisation d'un français moderne, standard, qui n'est plus celui de la littérature. La priorité est donnée à l'oral et à l'expression de l'élève. Les trois textes qui sont à l'origine de la rénovation du français à l'école élémentaire, Le projet Rouchette, le Plan de rénovation et les instructions de 1972, introduisent donc deux modalités dans l'enseignement de la langue : la communication et l'expression. L'élément central est la communication, puisque : "L'enseignement du français est donné pour aider l'enfant à communiquer et à penser " ${ }^{(28)}$. Vient ensuite l'expression qui est l'objectif de tout apprentissage linguistique : "Tous les travaux de rédaction à l'école, du plus modeste au plus ambitieux, ont pour but de munir l'élève des moyens de l'expression écrite et de le rendre habile à s'en servir dans toutes les circonstances où il sera porté à le faire par obligation ou par goût ${ }^{(29)}$. Pour souligner cette double finalité, la rédaction est officiellement qualifiée d' " expression écrite » et les écrits d'expression personnelle vont occuper une place particulière dans le dispositif de libération qui précède tout apprentissage ainsi que dans les recommandations officielles de 1972: "Mais les grandes sources de sujets sont les travaux de la classe; collectifs et individuels, actuels, passés ou en projet [...] ; ce sont les activités et distractions hors de l'école, les congés, les vacances, les voyages, les fêtes, les spectacles, les émissions de télévision ; [...] bref, tout ce qui est vécu ou imaginé par les enfants. » (I.O., p. 229).

Dans ce nouveau contexte, l'écriture est envisagée sous l'angle de l'expression

(25) INRP (Institut national de la recherche pédagogique) change de nom au cours de son histoire. Créé en 1956 sous le nom d'IPN (Institut pédagogique national), il devient INRDP en 1970 (Institut national de la recherche et de la documentation pédagogiques) et INRP en 1976. Il sera désigné sous le titre d'INRP tout au long de l'article.

(26) L'Union nationale inter-universitaire, association créée en réactions aux événements de 1968.

(27) Jean-François Halté remet en question la reconfiguration à la suite de la rénovation : «Le français entre rénovation et reconfiguration ", article repris dans Pratiques $n^{\circ}$ 137/138, juin 2008, pp. 23-38.

(28) « 4 décembre 1972 - Instructions officielles », dans A. Chervel, L'enseignement du français à l'école primaire, op. cit., p. 211.

(29) Instructions de 1972, in André Chervel, op. cit., p. 226. 
de soi et la communication est plus le cadre externe de la production que son fondement, contrairement à la pédagogie Freinet.

Ces conceptions se retrouvent dans les manuels édités à la suite de la rénovation où les sujets sollicitant le vécu ou l'expérience des élèves sont nombreux. Ainsi, dans un ouvrage de cours moyen publié par Larousse en $1978^{(30)}$, la moitié des sujets de rédaction est de type autobiographique. Il s'agit souvent de faire référence à des éléments du quotidien ou de la vie personnelle : "Décris ton meilleur copain." ou d'exprimer des goûts ou des préférences : "Quel métier aimerais-tu faire plus tard? Pourquoi? Si tu n'as pas encore d'idées, essaie quand de dire quel genre de métier te plairait le plus ». L'expression des sentiments y occupe aussi une grande place, toujours en relation avec la vie personnelle : "Il y a sans doute un sport que tu préfères. Tu regardes les épreuves à la télévision, tu rêves de le pratiquer un jour. Tu le pratiques déjà. Dis en quelques lignes quel est ce sport et pourquoi tu l'aimes. "

Ces rédactions présentent des caractéristiques nouvelles. Tout d'abord, mai 68 est passé par là et le « tu » injonctif a remplacé le « vous ». Le monde évoqué est plus moderne, on y rencontre les sports, la télévision, les voyages, etc. Mais surtout, les auteurs tentent de motiver l'écriture des élèves en introduisant des alternatives dans leurs intitulés de devoirs, s'assurant ainsi de la validité du souvenir évoqué : "Si tu as déjà assisté à une représentation de cirque, raconte le ou les numéros qui t'ont le plus passionnés, fait rire ou frémir. "

La tendance est de transformer la rédaction en une véritable expression personnelle, authentique pour laquelle on demande de faire preuve de sincérité : «Est-ce que tu as eu peur de l'orage? Réponds bien franchement et explique ce qu'il faut faire en temps d'orage."

Le principe d'une écriture de soi scolaire authentique et sincère s'installe à la suite de la rénovation. On en en retrouve les principes dans les travaux des élèves qui sont fréquemment des textes libres.

\section{Les textes libres de la rénovation}

Les textes libres sont recommandés par les instructions de 1972 pour faciliter la communication et surtout l'expression :

"En ce qui concerne le texte libre authentique, précisons qu'il a des caractères propres qui le distinguent sensiblement de la rédaction à sujet libre. Sa fonction essentielle est de faciliter la communication au moyen de l'écriture, et en même temps de faire bénéficier la langue écrite du même élan naturel et spontané que la langue parlée. Non seulement, l'enfant choisit le sujet, mais il choisit aussi le moment; il produit le texte quand il le souhaite, de sa propre initiative, sans être obligé de le montrer ensuite au maître. » (I.O., p. 227).

Mais contrairement aux textes libres des classes Freinet, les écrits des élèves ne sont pas systématiquement destinés à être diffusés. Leur rôle est la libération de l'expression, à ce titre, ils peuvent rester privés. Il est d'ailleurs conseillé aux maîtres de les recevoir avec discrétion et doigté, car l'élève peut y avoir " apporté sur des tiers ou sur sa famille des révélations pénibles " ${ }^{(31)}$. Le vécu rapporté peut être sensible, voire douloureux pour l'élève, dans tous les cas, il a cessé d'être anodin, car il s'agit d'un vécu authentique. La sincérité des écrits est essentielle, elle joue un rôle certain dans la démarche ternaire d'acquisition de la langue, libération / struc-

(30) Lecture et expression, CM1, Roland Eluerd, Albert Faget, Gérard Fenneteau, Paris, Larousse, 1978

(31) Instructions de 1972, in A. Chervel, op. cit., p. 229. 
turation / maîtrise, développée par le Plan de rénovation. Après le premier temps de libération de la parole, le maitre propose des exercices de structuration fondés sur le maniement, puis l'observation des structures linguistiques. Ce second temps permet l'acquisition de formes grammaticales et lexicales, plus complexes et adaptées aux différentes situations de communication. Le troisième moment est celui de l'utilisation consciente et maîtrisée de la communication orale et écrite, il s'agit d'un temps de synthèse et de transfert. Cette troisième étape constitue un retour à l'expression personnelle, maîtrisée car enrichie des savoirs de la seconde phase. Cette démarche ternaire repose sur deux principes. Le premier est que le désir de s'exprimer est naturel et qu'il suffit de libérer la parole pour la faire jaillir. Le second est que tout enfant est naturellement créatif, principe qui occupe une place importante dans le Plan. Cette conception de l'expression est fondée sur la spontanéité et sur une vision assez romantique de l'individu que nous pouvons voir à l'œuvre dans les travaux d'élèves. Mais faute d'un corpus conséquent, il n'est pas aisé de connaître ce qu'ont écrit les élèves entre 1972 et 1985. Dans les cahiers de cette période figurent assez peu de rédactions, ce qui peut s'expliquer par des changements de supports : les classeurs et leurs feuilles «volantes » remplaçant quelques fois les cahiers traditionnels. Mais ce phénomène de raréfaction est également lié aux modifications importantes que connaissent les écritures scolaires. En effet, le changement de modalité d'écriture a sorti les travaux de rédaction des cahiers, les textes libres, les correspondances interclasse, les enquêtes ou les poèmes se trouvant sur des affiches, dans des journaux scolaires, quelques fois dans des cahiers ou classeurs « d'enquêtes ». Mais si le corpus est réduit à une quinzaine de textes libres de type autobiographique, écrits entre 1972 et 1985, on y remarque des éléments récurrents. Le premier de ces traits est la forte centration du texte sur le narrateur, comme dans ce récit rédigé par un élève de CM2, au cours de l'année scolaire 1974-1975 :

"Ma première leçon d'équitation:

Un jour que je souhaitais faire du cheval, mon papa m'a inscrit à la société hippique de Pomméréval. Le jour venu je me rendis au manège. Le cercle hippique est situé dans une grande cour de ferme. Autour il y a une grange, des écuries, un bâtiment ouvert pour ferrer les chevaux et une maison dans laquelle se trouvait le bureau. La cour s'ouvrait sur une grande prairie où galopent des chevaux en liberté. Le moniteur me désigna mon cheval. Il s'appelle "Où vas-tu" et celui de ma sour "Napoléon". Je prends mon cheval par la bride et l'emmène dans le grand bâtiment qui abrite le manège.

Le moniteur m'apprit à monter en selle puis à marcher au pas et au trot. Je suis satisfait de cette première leçon et Sophie aussi.»

L'expérience relatée est rigoureusement personnelle et c'est donc autour de l'événement vécu que s'organise le récit. La seconde caractéristique de ces textes est leur organisation chronologique, il s'agit souvent d'une énumération d'actions, comme dans ce devoir rédigé en mars 1971 par un élève de CM2 :

"Jeux dans la neige,

Lorsque je suis sortie de l'école il neigeait un peu, puis ça s'arrêtait mais quand je suis allée en classe il recommençait à neiger encore plus fort que tout à l'heure. Enfin, c'était l'heure de la récréation, quand nous sommes sortis, déjà les glissades commençait il y en avait qui jouait aux boules de neige, d'autres qui restaient dans un coin parce qu'ils ne voulaient pas jouer dans la neige assez froide, mais la récréation est passée et il est l'heure de rentrer. Ils recommenceront certainement cet après-midi...»

Ces textes libres de la rénovation ont une fonction libératoire puisqu'ils doivent motiver le désir d'écrire, sans autre finalité que l'expression de l'individu. Leurs 
enjeux sont très différents de ceux des textes libres de la pédagogie Freinet. Chez ce dernier, les écritures de soi sont destinées à être socialisées, elles ne peuvent se centrer sur l'individu, tandis que les écritures de soi de la période de la rénovation sont essentiellement expressives, c'est-à-dire qu'elles demeurent dans la sphère individuelle, qui en constitue le mobile et la finalité. Elles contribuent ainsi à la libération de la parole. Paradoxalement, cette liberté acquise dans le modèle de la rénovation s'accompagne de ce qui ressemble à un appauvrissement des textes produits, ce qui va provoquer nombre de critiques, dès les années soixante-quinze, c'est-à-dire au moment de la réforme Haby.

\section{Le déclin des écritures de soi}

\section{La didactique de l'écriture}

La rénovation transforme non seulement la configuration disciplinaire du français mais également les modalités de réflexion pédagogiques. L'expérimentation et le travail des équipes de l'INRP introduisent le concept de « recherche-action » qui donne naissance à la didactique. Cependant le mouvement réformateur semble rencontrer des résistances et la période qui commence au début des années quatre-vingt est celle d'un retour à une conception plus traditionnelle de l'enseignement élémentaire. Plusieurs causes peuvent être évoquées. La première est sans doute le désir des politiques et d'une partie de la société, face à la crainte suscitée par l'illettrisme, de revenir à un apprentissage de la langue moins libéral, recentré sur les savoirs fondamentaux. Les instructions de 1985 vont dans ce sens, en proposant un retour au «lire-écrire-compter». La seconde cause est liée à la réforme elle-même. Trop complexe, elle est difficile à mettre en œuvre pour des maîtres insuffisamment formés. Souvent ils n'en gardent qu'une partie, la plus aisée à transposer dans leur classe, négligeant l'ensemble de la démarche qu'ils ne maîtrisent pas. C'est ce qui se produit avec le texte libre qui est utilisé sans être suivi de la phase de structuration et d'apprentissage de la langue. Enfin, la troisième cause, liée aux deux précédentes, est celle d'un sentiment partagé aux différents niveaux scolaires et dans la société d'une baisse du niveau des connaissances au sortir de l'école élémentaire. Pour en déterminer les causes, le ministre de l'éducation nationale de la gauche récemment arrivée au pouvoir, Alain Savary, confie, en 1983, à une commission présidée par le linguiste Jean-Claude Chevalier, le soin de mener une réflexion sur l'enseignement du français. Une partie des conclusions concerne le lire et écrire à l'école élémentaire, certaines sont sévères. Tout d'abord il apparaît que le texte libre est devenue l'une des principales activités d'expression écrite mises en œuvre dans les classes élémentaires. Mais cette pratique est jugée inapte à favoriser un enseignement de l'écriture, par les membres de la commission. Selon leur analyse, le texte libre détourne l'attention de ce qui est primordial, c'est-à-dire des procédures des élèves et conduit les maîtres à apporter des réponses inadaptées aux problèmes rencontrés, allant même jusqu'à abandonner toute exigence rédactionnelle :

« [Les maîtres] s'enferment dans des situations impossibles en imposant régulièrement d'écrire des "textes libres", sans la moindre précision, ou en ne proposant qu'une consigne thématique qui fait appel au vécu des enfants ("racontez vos dernières vacances"). Devant les difficultés des élèves, ils cherchent à leur simplifier la tâche : "Faites des phrases courtes", "N'écrivez qu'un paragraphe", "Mettez tout au présent" ${ }^{(32)}$."

(32) Réflexions sur l'enseignement du français 1983-1985, Rapport de la Commission de Réflexion sur 1'Enseignement du français, Angers, CNDP de Maine et Loire, 1985, p. 174. 
Le constat est sans appel, le texte libre masque les problèmes réels derrière la liberté d'expression, l'authenticité et la spontanéité. L'écriture est trop souvent envisagée comme une pratique qui ne s'enseigne pas et les maîtres ignorent les moyens à mettre en œuvre pour en faciliter l'apprentissage. Les membres de la commission proposent de prendre appui sur les travaux naissant de la didactique de l'écriture. Il s'agit d'une part des apports des théories textuelles et de ceux de la psychologie cognitive. Ces nouvelles approches vont permettre d'élaborer des conceptions d'apprentissage de l'écriture, fondées sur les concepts de procédures et de types de textes. Les équipes de l'INRP et les différentes revues, Pratiques, Repères et le Français aujourd'hui s'emparent de ces questions et s'efforcent de mettre en relation les apports de la linguistique et les démarches d'enseignement. En 1986, la didactique va naître officiellement de ces travaux avec la création de la DFLM (Association internationale pour le Développement de la Recherche en Didactique du Français Langue Maternelle) à Namur. L'ensemble des propositions possède certains traits communs, elles s'organisent autour de trois pivots qui sont largement développés, en 1992, dans La maîtrise de la langue. Il s'agit de l'organisation textuelle, de la réécriture associée à la maîtrise du brouillon et de l'évaluation. Mais les écritures de soi peinent à y trouver leur place, elles n'appartiennent à aucun type de textes, elles résistent aux procédures d'évaluation et la réécriture fait mauvais ménage avec le principe de spontanéité et de sincérité auquel elles sont associées depuis la rénovation.

\section{De nouveaux usages de l'écriture subjective}

Les exercices scolaires naissent, se développent, se transforment et meurent. C'est le cas des écritures de soi qui disparaissent des textes officiels, à partir de 1985. Cela commence par l'effacement de toute référence au texte libre à partir de 1980, dans les programmes du cycle moyen. Puis, dans les instructions de 1985, il n'est plus fait appel au vécu pour susciter l'envie d'écrire. Au contraire, les apports d'une réflexion sur un apprentissage structuré de l'écriture font leur apparition, puisqu' on y insiste sur la nécessité de travailler différents genres, surtout les récits, et l'accent y est mis sur la composition des textes : «plan, articulation des idées, organisation en paragraphes ${ }^{(33)} »$. Dans tous les cas, l'écrit doit être repris, corrigé et amélioré pour favoriser un véritable apprentissage. On passe de la représentation de l'élève détenteur d'une histoire sur laquelle on peut prendre appui à celle d'un élève scripteur, c'est-à-dire que l'intérêt se centre sur l'activité de production de texte qu'il faut faciliter par la prise en compte des différentes procédures.

Mais dans les manuels, les écritures de soi sont encore présentes jusqu'aux années quatre-vingt-dix, comme si, dans les écoles, malgré la prescription, la référence au vécu restait un moyen privilégié de faire écrire les élèves. Ainsi, dans un manuel publié en 1987 chez Bordas ${ }^{(34)}$, se retrouvent les mêmes sujets que ceux de la période précédente. Par exemple, il est demandé de raconter des faits ou de faire référence à des éléments de la vie familiale :

«Décris ceux que tu aimes. Essaie de raconter l'un des sujets suivants :- ce qui se passe tous les soirs après la sortie de l'école; - ce qui se passe tous les soirs après le dîner ; - ce qui se passe à la maison tous les dimanches matins. »

Curieusement, le récit de la soirée en famille, souvenir de la première période re-

(33) École élémentaire, Programmes..., op. cit., p. 34.

(34) Français CM1 de Gilbert PY, 1987, Paris, Bordas 
paraît dans cet intitulé, mais avec un attendu différent, puisqu'on demande à l'élève une évocation authentique. Les demandes peuvent également porter sur les goûts ou sur les opinions :

«Parmi tous les métiers que tu connais quel est celui que tu aimerais faire ? Explique ton choix en donnant les avantages et les inconvénients. »

Ces propositions qui ne diffèrent pas de celles de la période de la rénovation sont courantes, elles représentent largement plus de la moitié des sujets de rédaction des différents manuels consultés entre 1987 et 1989.

Finalement, c'est à la suite de la diffusion par La maîtrise de la langue en 1992 des approches didactiques de l'écriture, reprises de manière succincte dans les instructions de 1995, que ces sujets vont devenir plus rares dans les manuels. Il s'agit bien d'un déclin, car les écritures de soi paraissent en partie résistantes à l'apprentissage explicite et procédural, de plus, elles sont rarement perçues comme un genre scolaire enseignable.

Toutefois, deux tendances apparaissent dans des manuels plus récents. L'une est une approche générique des écritures de soi. C'est le cas de l'ouvrage de Renée Léon ${ }^{(35)}$, publié chez Hachette en 2004, dans lequel l'auteure aborde le récit d'événement vécu comme un genre de texte particulier que on peut apprendre à construire. Cette démarche avait déjà été proposée en 1996 par les éditions Nathan, dans l'ouvrage de Bernard Scheuwly et Jean-Michel Sandon ${ }^{(36)}$. La seconde tendance est à rapprocher des évolutions de la lecture littéraire à l'école élémentaire. Pour aider les élèves à s'approprier les textes par une lecture personnelle et interprétative, on prend appui sur l'écriture personnelle, qui peut être une demande de justification ou l'explication d'une opinion, comme dans ce manuel de $2003^{\left({ }^{(37)} \text { édité }\right.}$ chez Magnard :

«Regarde à nouveau la couverture du livre, p. 100. Trouves-tu que le titre est bien choisi ? que l'illustration est bien choisie ? que la mention "policier" est bien choisie ? Si vous avez répondu "non" à l'une de ces questions, écrivez une lettre que vous enverrez à l'éditeur pour lui dire pourquoi vous n'êtes pas d'accord et lui demander de lui expliquer vos choix. Voici son adresse ...» (2003, p. 123)

La sollicitation de la subjectivité occupe une nouvelle place dans l'appropriation du sens des textes en ce qu'elle rend compte des démarches interprétatives des lecteurs, la lecture littéraire devenant une occasion de retrouver la voix personnelle de l'élève ${ }^{(38)}$.

Néanmoins, une dernière voie demeure, celle qui consiste à utiliser le vécu comme prétexte et matière pour évaluer la langue et apprendre la grammaire. C'est le cas de certaines propositions, généralement dans le domaine de l'étude de langue. Ainsi, pour employer le passé composé, un manuel de 2004 demande aux élèves de tenir un journal de bord: "Pendant quelques jours, écris un journal de bord, en fin de journée, pour raconter ce qui s'est passé, ce qui t'a intéressé, ce qui t'a marqué, ce que tu as pensé... » Ou encore d'envisager leur futur métier en utilisant

(35) Renée Léon, Français, des outils pour lire, dire et écrire, CM1, Paris : Hachette, 2004,

(36) Bernard Scheuwly et Jean-Michel Sandon, Expression écrite, CE2, Guide pédagogique, Paris, Nathan, 1996.

(37) Brigitte Louichon et Elisabeth Grimaldi, Ricochets, CM1, Paris : Magnard, 2003.

(38) Voir l'analyse d'Yves Reuter, qui considère la littérature comme un médiateur permettant 1'expression de la subjectivité des élèves, dans «Éléments de réflexion sur la place et les fonctions de la littérature », dans Repères, n¹3, 1996, pp. 7-25. 
le futur: "Imagine ce que tu feras dans vingt ou trente ans. Écris un petit texte. Emploie des verbes au futur. " Nous retrouvons dans ces propositions l'ambiguïté inhérente aux écritures de soi, qui sont souvent perçues comme spontanées, «faciles », alors qu'elles nécessitent un véritable travail d'écriture et de mise à distance du vécu pour en faire un récit. Le paradoxe des écritures de soi scolaires qui ne s'enseignent pas, ne se construisent pas, ne se corrigent pas, risque de resurgir dans ce type d'exercice.

\section{Conclusion}

L'intérêt de la perspective historique a été de souligner en quoi l'usage des écritures de soi à l'école dévoile les finalités qui président à la scolarisation des individus. Dans le modèle traditionnel, ce dernier est avant tout un sujet scolaire et social à éduquer et à transformer par l'acquisition de schémas linguistiques et moraux. Tandis que chez Freinet, l'élève apparaît comme un sujet collectif et politique qui s'épanouit lorsque sa nature profonde est respectée. La rénovation, marquée par 1'idéologie de mai 68, a eu tendance à accorder la primauté à l'expression individuelle, au détriment des repères sociaux, dans une perspective libératoire. Actuellement, les écritures de soi subissent deux influences contradictoires. D'une part, elles sont refoulées hors de l'école par le souci de plus en plus affirmé du respect des limites entre le privé et le social. D' autre part, on assiste, dans le champ scolaire, à l'apparition de formes proches qui sans être des écritures de soi en partagent certaines caractéristiques, ce sont les écritures réflexives, qui coïncident avec la tendance de nos sociétés à valoriser un individu réflexif, capable d'agir et d'analyser son action.

Pour finir, en revenant sur l'histoire de cet exercice de rédaction bien particulier, il a été possible de s'interroger sur la démarche historique au sein de la didactique. En effet, si cette approche est extrêmement heuristique elle est aussi problématique par certains aspects. D'abord, en ce qu'elle croise différentes disciplines de recherche qui sont l'histoire, la sociologie et parfois l'analyse politique, c'est-à-dire des éléments externes, pour répondre à des questions internes de didactique, qui reste la discipline de référence. Ensuite, en ce qu'elle s'appuie sur une diversité de documents et d'ouvrages, de statut très différent, sans que l'origine des différents discours soit facilement identifiable. Enfin, en ce que son hybridité la fragilise, la rendant toujours incomplète et discutable, du fait de son manque de méthodologie propre. En cela, la perspective « didactico-historique» (pour oser ce barbarisme !) est sans doute l'une des voies qui peine le plus à trouver sa légitimité au sein d'un champ déjà en mal de reconnaissance, celui de la didactique du français. 


\section{Bibliographie}

BISHOP, M.-F. (2004) : Les écritures de soi à l'école primaire de 1850 à 2004, Thèse de doctorat, Lille3.

Boutan, P.(1996) : La langue des Messieurs. Histoire de l'enseignement du français à l'école primaire, Paris, Armand Colin.

CABAnel, P. (2002) : La République du certificat d'études, Paris : Belin.

Chanfrault-Duchet, M.-F. (2006) : «Subjectivité et apprentissage de l'écriture au collège : pratiques et enjeux ", dans Repères, $n^{\circ} 34, L^{\prime}$ 'écriture de soi et l'école, M.-F. Bishop et M.-C. Penloup, coord. p.85-109.

Chervel, A. et MANesse, D., (1989) : La dictée, les Français et l'orthographe (1873-1987), Paris, Calman-Lévy.

Chervel, A. (1988) : «Pour une histoire des disciplines scolaires », Histoire de l'éducation, $\mathrm{n}^{\circ} 38$, pp. 59-119.

— (1995): L'enseignement du français à l'école primaire. T.1, 2, 3 Paris : INRP.

- (2006) : Histoire de l'enseignement du français du XVII au XX $X^{e}$ siècle, Paris, Retz.

Deloye, Y. (1994) : École et citoyenneté, Paris, Presses de la Fondation nationale des Sciences politiques.

DENIZOT, N. (2008) : Genres littéraires et genres textuels en classe de français. Thèse de doctorat, Lille 3.

Dubet, F. (2002) : Le déclin de l'institution, Paris : éd. Du Seuil.

HOUDART-MÉROT, V., (1998) : La culture littéraire au lycée depuis 1880, Paris/Rennes, ADAPT-PUR.

JAUss, H.R, (1972 ; 1978, trad. française) : Pour une esthétique de la réception, Paris : Gallimard.

JEY, M., (1998) : La littérature au lycée : Invention d'une discipline (1880-1925), Recherches textuelles $n^{\circ} 3$, Metz, Université de Metz.

LABOV, W, (1978; 1993, trad. française) : Le parler ordinaire, Paris : éditions de Minuit.

LAHIRE, B. (2005) : L'esprit sociologique, Paris : éd. de la Découverte.

LEJEUnE, P. (1975) : Le pacte autobiographique, Paris : éd. du Seuil.

Martucelli, D. (2002) : Grammaires de l'individu, Paris, Gallimard.

PENLOUP, M.-C. (1999) : l'écriture extrascolaire des collégiens, Villeneuve d'Ascq, ESF.

Prost, A. (1968) : Histoire de l'enseignement en France 1800-1967, Paris : Armand Colin.

— (1985) : «Frontières et espaces du privé », dans Histoire de la vie privée, Dir. Philippe Ariès et Georges Duby, Paris : Seuil. pp. 13-132.

- (1992) : Éducation, société et politique, Paris, Seuil.

REUTER, Y, (2006) : «Le récit sollicitant le vécu au CM2 », dans Repères, $n^{\circ} 34$, $L^{\prime}$ 'écriture de soi et l'école, M.-F. Bishop et M.-C. Penloup, coord., pp. 113-139.

VINCENT, G. (1980) : L'école primaire française, Lyon : Presses Universitaires de Lyon.

VOURZAY, M.-H. (1996) : Cinq discours sur la rédaction (1870-1989), thèse pour le doctorat, université de Lyon. 